\title{
Wideband Signal Based Near-Field Electromagnetic Ranging for Indoor Localization
}

\author{
Peng Wang, Zhiyang Liu, Xiaotong Zhang*, Liyuan Xu, Jie He and Yadong Wan \\ School of Computer and Communication Engineering, University of Science and Technology Beijing, 30th Xueyuan Rode, \\ Haidian District, Beijing, 100083, China \\ *Corresponding author
}

\begin{abstract}
The near-field electromagnetic ranging (NFER) technology exploits the near-field phase behavior of low frequency signals to measure the distance and can provide better ranging performance than the high frequency signal based methods in the cluttered environments. However, the currently existing NFER system uses the mono-frequency signals and can not obtain same ranging accuracy at different distances. In addition, low signal-to-noise ratio (SNR) seriously impacts the ranging accuracy. In this paper, we proposed a wideband signal based NFER system to improve the ranging performance. Instead of the mono-frequency signals, the proposed wideband system transmits the wideband signals. We use frequency domain phase measurement (FDPM) to detect the phase difference between received electric field signal and magnetic field signal. Then the least squares method is used to estimate the distance. Simulation results show that the proposed wideband NFER system can improve the ranging performance at low SNR environment and within a wide range of distances.
\end{abstract}

Keywords- near-field electromagnetic ranging; wideband signal; frequency domain phase measurement; phase difference; least squares

\section{INTRODUCTION}

In the cluttered environments, such as warehouses, factories, underground mine, and so on, the high frequency signals are blocked by objects, causing big ranging error in the high frequency signal based ranging methods [1]. Therefore, the time of arrival (TOA), time difference of arrival (TDOA) and angle of arrival (AOA) methods based on high frequency signal can not get high positioning accuracy in Non-line-ofsight (NLOS) environments. The non-geometric approaches such as fingerprinting tracking algorithms exploit the received signal strength (RSS) based on a training database and are not suitable for the unknown environments [2]. Exploiting the near-field phase behavior of low frequency signals, typically within the AM broadcast band $(530-1710 \mathrm{kHz})$, the near-field electromagnetic ranging (NFER) technology can provide better ranging performance in the cluttered environments [3]. The low frequency signals have less path loss after penetrating structures and are less prone to multipath environment.

The currently existing NFER system, designed by Hans Schantz, transmits the mono-frequency signals and detects the phase difference between the received electric field and received magnetic field [4]. The NFER system does not require precise time synchronization between transmitters and receivers while the ranging accuracy just depends on the precise phase measurements between electric field channel and magnetic field channel. Based on the monotone lowfrequency signals (typically around $1 \mathrm{MHz}$ ), the NFER system is limited to short ranging distance $(30-65 \mathrm{~m})$ and can not obtain same ranging accuracy at different distances. In the near field, the signal strength suffers greater attenuation compared to the far field and the noise intensity is higher in the low frequency band [5]. Therefore, the low signal-to-noise ratio (SNR) seriously impacts the ranging accuracy of NFER system when the ranging distance is longer than $25 \mathrm{~m}$ [6]. In order to improve the ranging accuracy, multiple frequencies approach is proposed in [7]. But the transmitted signal is in the form of a stepped frequencies signal, which increases the complexity of NFER system and increases the processing time, not suitable for real-time location system.

In this paper, we proposed a wideband signal based NFER system to improve the ranging performance. Instead of the mono-frequency signals, the proposed wideband system transmits the wideband signals, such as the second-order derivative of the Gaussian pulse signal, multisines, and so on. After sampling, the received electric field signal and magnetic field signal are processed by frequency domain phase measurement (FDPM) to get the phase differences at multiple frequencies. Then the least squares method is used to estimate the distance. Simulation results show that the proposed wideband NFER system can greatly improve the ranging performance.

The remainder of this paper is organized as follows. Section II describes the proposed wideband NFER system. Section III evaluates the performance of the proposed system. We present conclusions in Section IV.

\section{WIDEBAND NEAR-FIELD ELECTROMAGNETIC RANGING}

\section{A. Near-Field Electromagnetic Ranging}

Consider a Hertzian electric dipole with length $l$ and current $I$, the phase relationships of the electric and magnetic field are

$$
\begin{gathered}
\varphi_{E}=-\frac{180}{\pi}\left\{\frac{\omega r}{c}+\left[\cot ^{-1}\left(\frac{\omega r}{c}-\frac{c}{r \omega}\right)+n \pi\right]\right\} \\
\varphi_{\mathrm{H}}=-\frac{180}{\pi}\left[\frac{\omega r}{c}+\left(\cot ^{-1} \frac{\omega r}{c}+n \pi\right)\right]
\end{gathered}
$$


where, $\omega$ is the signal frequency, $r$ is the distance from the dipole, and $c$ is the speed of light. Then the phase difference between electric filed and magnetic field is

$$
\Delta \varphi=\cot ^{-1}\left(\frac{\omega r}{c}-\frac{c}{\omega r}\right)-\cot ^{-1} \frac{\omega r}{c}
$$

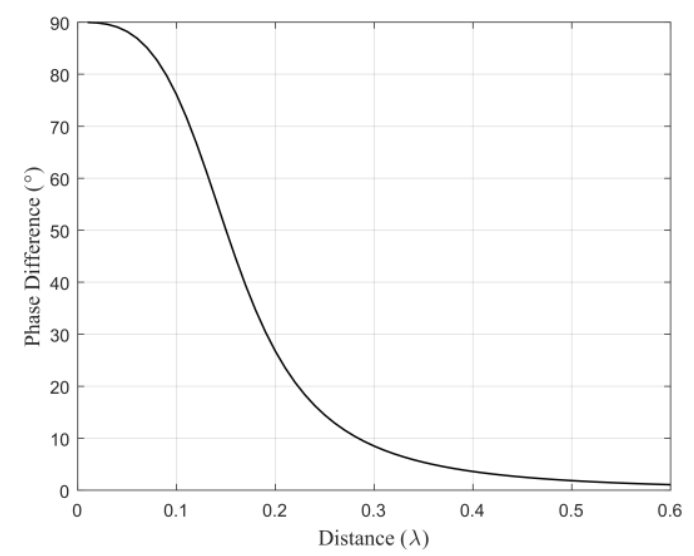

FIGURE I. THE RELATION BETWEEN DISTANCE $r$ AND PHASE DIFFERENCE $\Delta \Phi$.

The relationship between distance $r$ and phase difference $\Delta \varphi$ is

$$
r=\frac{\lambda}{2 \pi} \sqrt[3]{\cot \Delta \varphi}
$$

where, $\lambda$ is the wavelength of the electromagnetic signal. Figure I shows how the phase difference varies according to the distance. As shown in Figure I, the ranging performance is different at different distance when the phase difference detection accuracy is the same. The good ranging accuracy can only be achieved at the distance $r \in(0.08 \lambda, 0.25 \lambda)$ [7]. Therefore, using single frequency would limit the ranging accuracy. Multiple frequencies approach can improve the ranging accuracy, but also increases the complexity of NFER system. In this paper, we propose a wide-band NFER system, exploiting the wideband pulse as the transmitted signal to improve the ranging accuracy.

\section{B. Wideband NFER System}

Figure II shows the detail of the proposed wideband NFER system. The proposed system includes two part: a transmitter and a receiver. The wideband signal, such as a pulse, is generated by the signal generator and transmitted by antenna, which can be magnetic antenna or electric antenna. Then, the transmitted signal is received by magnetic field antenna and electric field antenna simultaneously. After analog-to-digital conversion (ADC), the sampling signal is processed by the digital part. To obtain the phase at a specific frequency, we use

the FDPM to get the phase behavior of the transmitted signal in a few specific frequencies. Then, we get the phase differences between the electric and magnetic field $\left(\Delta \varphi_{1}, \Delta \varphi_{2}, \mathrm{~K}, \Delta \varphi_{n}\right)$

in a few specific frequencies $\left(f_{1}, f_{2}, \mathrm{~K}, f_{n}\right) . f_{n}$ is the frequency contained within the signal bandwidth. With the signal frequencies and phase differences, we can estimate the distance according to (4). Next, we will describe the proposed wideband NFER system in detail.

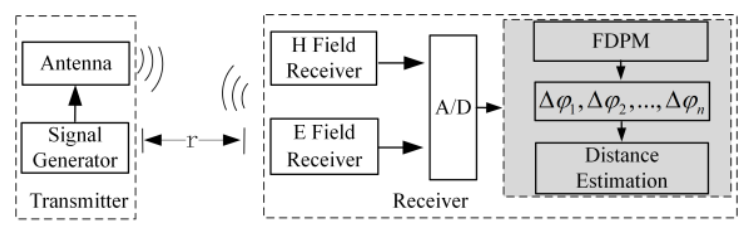

FIGURE II. THE WIDEBAND NFER SYSTEM

1) Signal Generation: In order to improve the ranging accuracy, the wideband signal should contain multiplefrequency signals [7]. Now, the signal frequency is limited to the amplitude modulation (AM) broadcasting band (510$1705 \mathrm{kHz}$ ). To get more accuracy ranging performance, we extend the signal frequency to $(0.5-10) \mathrm{MHz}$ and limit the transmit power to $100 \mathrm{~mW}$. There are many ways to generate the wideband signal. In this paper, we use the second-order derivative of the Gaussian pulse and multisines as the wideband signal respectively, to study the effect of signal shape on ranging performance. The second-order derivative of the Gaussian pulse signal $s_{G}$ can be presented as [8]

$$
S_{G}(t)=E_{p}\left[1-4 \pi\left(\frac{t-t_{c}}{T_{p}}\right)^{2}\right] e^{-2 \pi\left(\frac{t-t_{c}}{T_{p}}\right)^{2}}
$$

where, $E_{p}$ is the pulse amplitude, $T_{p}$ is a pulse width adjustment factor, $t_{c}$ is a time shifting of the pulse. A typical multisine signal $s_{M}$ can be presented as [9]

$$
S_{M}(t)=\sum_{k=1}^{N} A_{k} \cos \left(\omega_{k} t+\theta_{k}\right)
$$

where, $A_{k}$ and $\theta_{k}$ are the amplitude and phase of the $k$ th sinusoid, $N$ is the number of sinusoids, and $\omega_{k}$ is angular frequency of the $k$ th sinusoid,

$$
\begin{aligned}
\omega_{k} & =\omega_{0}+(k-1) \Delta \omega \\
& =2 \pi\left[f_{0}+(k-1) \Delta f\right]
\end{aligned}
$$

where, $\omega_{0}$ and $f_{0}$ are the angular frequency and frequency of the first tone respectively, $\Delta \omega$ and $\Delta f$ are the constant frequency separations. By adjusting the parameters in (5) and (6), we can generate the wideband signal with frequency in (0.5-10) $\mathrm{MHz}$ band. 
2) Frequency Domain Phase Measurement: For a wideband signal, it is difficult to measure the phase for multiple frequencies in the time domain. Frequency domain measurement has been widely used for ultrawideband (UWB) signal processing in TOA estimation [10], doppler radar [11], synthetic aperture radar (SAR) [12] and channel modeling [13]. To measure the phase of sampled electric and magnetic signal, we can use the discrete Fourier transform (DFT) [14],

$$
F_{n}=\sum_{k=0}^{N} s(k) e^{-2 \pi i k n / N}=\operatorname{Re}_{n}+i \operatorname{Im}_{n}
$$

where, $i$ is the imaginary number, $\mathrm{Re}$ and $\mathrm{Im}$ are respectively the real and imaginary parts of the transform at frequency $f_{n}$. Then the phase $\varphi_{n}$ is obtained with

$$
\varphi_{n}=\arctan \left(\frac{\operatorname{Im}_{n}}{\operatorname{Re}_{n}}\right)
$$

By processing the electric signal and magnetic signal at frequency domain simultaneously, we can get the phase differences between the electric and magnetic field $\left(\Delta \varphi_{1}, \Delta \varphi_{2}, \mathrm{~K}, \Delta \varphi_{n}\right)$.

3) Distance Estimation: According to (4), the relationship between distance $r$ and phase difference $\Delta \varphi$ at signal frequency $f$ is

$$
\left(\frac{2 \pi r}{c} f\right)^{3}=\cot (\Delta \varphi)
$$

Substituting $\alpha=\left(\frac{2 \pi r}{c}\right)^{3}$ into(10), we get

$$
\alpha f^{3}=\cot (\Delta \varphi)
$$

Suppose that we get the phase differences $\Delta \varphi=\left[\Delta \varphi_{1}, \Delta \varphi_{2}, \mathrm{~K}, \Delta \varphi_{n}\right]^{\mathrm{T}} \quad$ at $\quad$ frequencies $\mathbf{f}=\left[f_{1}, f_{2}, \mathbf{K}, f_{n}\right]^{T}$, then

$$
\alpha \mathbf{f}^{3}=\cot (\Delta \varphi)+\mathbf{e}
$$

where, $\quad \mathbf{e}=\left[e_{1}, e_{2}, \ldots, e_{n}\right]^{T}$ is the phase difference measurement error, caused by indoor electromagnetic noise and frequency domain phase measurement. According to the least squares method [15], we can get the estimated value $\hat{\alpha}$ by

$$
\hat{\alpha}=\arg \min \left\{\left\|\alpha \mathbf{f}^{3}-\cot (\Delta \varphi)\right\|^{2}\right\}
$$

Then we get

$$
\hat{\alpha}=\frac{\sum_{i=1}^{N} f_{i}^{3} \cot \left(\Delta \varphi_{i}\right)}{\sum_{i=1}^{N} f_{i}^{6}}
$$

Therefore, the estimated distance is

$$
\hat{r}=\frac{c \sqrt[3]{\hat{\alpha}}}{2 \pi}
$$

\section{SimUlation Results}

To evaluate the ranging performance of proposed wideband NFER method, we build the simulation system with Matlab, as shown in Figure III. The signal generator can generate two kinds of signal with frequency from $1 \mathrm{MHZ}$ to 10 MHZ: the second-order derivative of the Gaussian pulse and multisines. The near field model is the relationship between distance $r$ and phase difference $\Delta \varphi$ in (4) and the transmitted signals are mixed with Gaussian noise. After sampled with $1 \mathrm{GHz}$ sampling rate, the transmitted signals are processed with 100-point fast Fourier transform (FFT). Then we calculate the phase difference between electric filed signal and magnetic signal at $(1,2, \ldots, 10) \mathrm{MHz}$ and use the least squares method to estimate the distance. Next, we will describe the simulation results in detail.

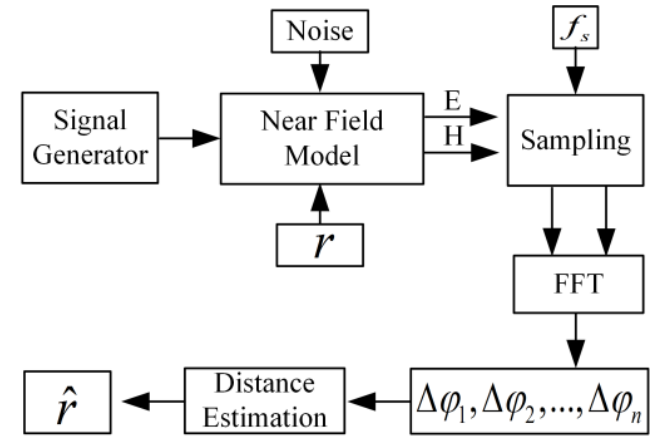

FIGURE III. THE WIDEBAND NFER SIMULATION DIAGRAM. 


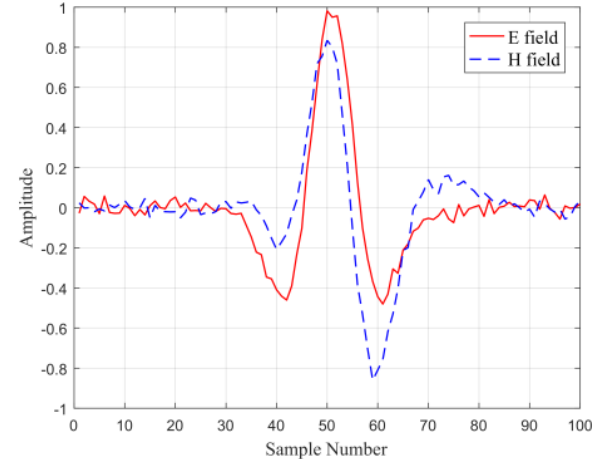

(a). The second-order derivative of Gaussian pulse

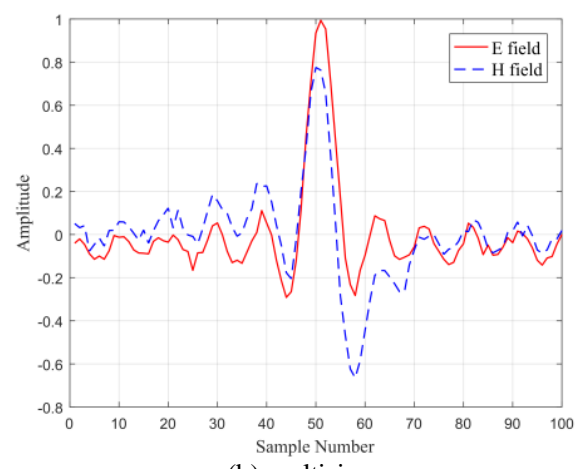

(b) multisines

FIGURE IV. THE RECEIVED WIDEBAND SIGNAL.

\section{A. Wideband Signal}

Figure IV shows the received signal after sampling when the distance $r$ is $15 \mathrm{~m}$ and SNR is $20 \mathrm{~dB}$. The shapes of the electric field signal and the magnetic field signal are different, because the phase differences at different frequencies are not the same. Both the second-order derivative of the Gaussian pulse and multisines can be seen as limited-width pulses in time domain, but the multisines have much heavier tails.

\section{B. Ranging Performance}

In order to improve the ranging performance, we collect 10 cycles of data and calculate the phase differences between electric filed signal and magnetic signal at $(1,2, \ldots, 10) \mathrm{MHz}$. Figure $\mathrm{V}$ shows the phase difference calculation results when the distance $r$ is $15 \mathrm{~m}$ and the SNR is $10 \mathrm{~dB}$. For the secondorder derivative of the Gaussian pulse, most of the signal power is in the center frequency band. Therefore, the center frequency band signal is less affected by the noise. As shown in Figure 5a, the phase difference calculation results at $(3,4$, $5,6) \mathrm{MHz}$ have less variance than those at other frequencies. For the multisines, the signal power at all frequencies is almost the same. Therefore, the variances of the phase difference calculation results at all frequencies are less affected by the noise. According to (12), the relationship between phase differences and frequencies at fixed distance is a curve. We show the estimated distance and real distance $(15 \mathrm{~m})$ in Figure V. With two kinds of wideband signal, we both can get the estimated distance with little error. The distance estimation mean error with the second-order derivative of the Gaussian pulse is $0.29 \mathrm{~m}$ and that with the multisines is $0.22 \mathrm{~m}$.

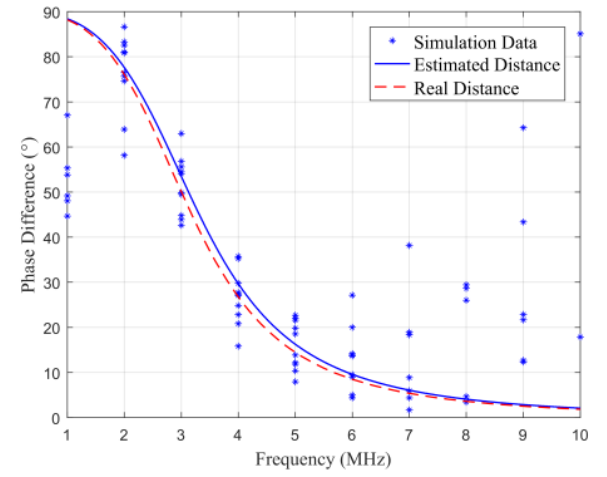

(a) The second-order derivative of the Gaussian pulse

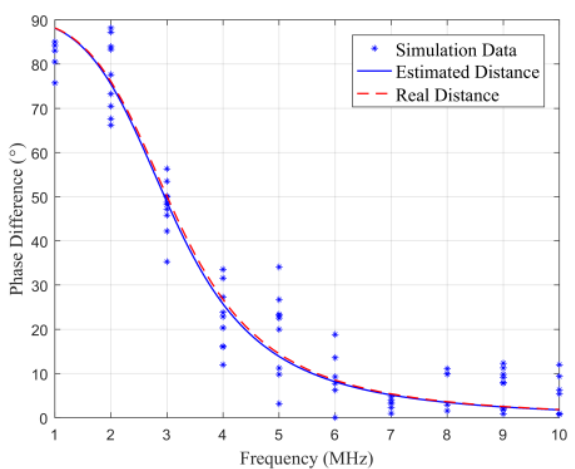

(b) multisines

FIGURE V. DISTANCE ESTIMATION RESULTS

To show the ranging performance improvement, we compare the ranging performance of proposed wideband method with that of the adaptive time delay estimation (ATDE) algorithm proposed in [16]. Figure VI(a) shows the ranging performance of three methods at different SNR when the distance is $15 \mathrm{~m}$. The proposed wideband NFER system can greatly improve the ranging performance at low SNR environment and the mean ranging error of proposed wideband NFER system is smaller than $0.1 \mathrm{~m}$ when SNR is higher than 20dB. Figure VI(b) shows the ranging performance of three methods at different distances when SNR is $20 \mathrm{~dB}$. The signal frequency used in ATDE method is $5 \mathrm{MHz}$. Therefore, the ATDE method can only achieve good ranging performance from $5 \mathrm{~m}$ to $20 \mathrm{~m}$. But the proposed wideband method can achieve good ranging performance in the whole range. In Figure VII., we compare the ranging performance of three methods at two dimension (SNR and distance). The proposed wideband NFER method can achieve better ranging performance than the narrowband ATDE method. Therefore, the proposed wideband NFER method can improve the ranging performance. 


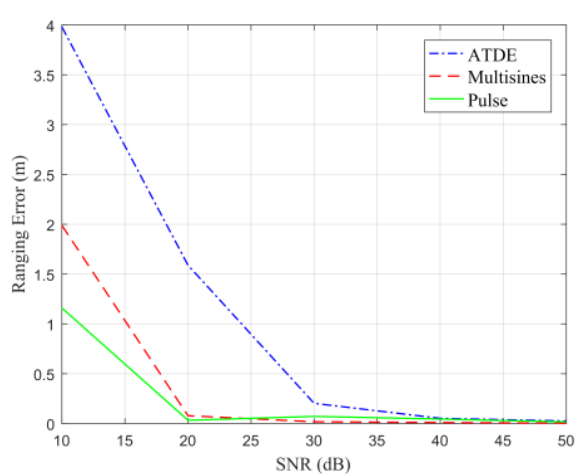

(a) Ranging performance at different SNR

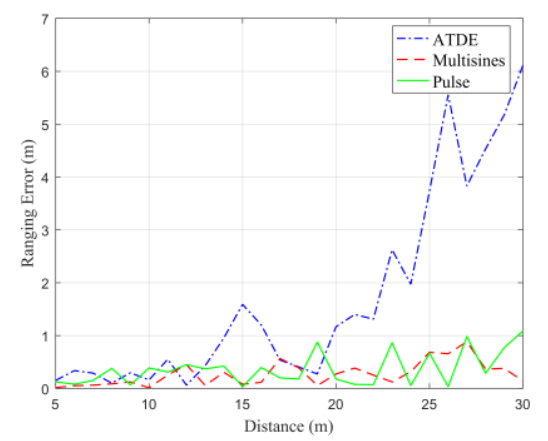

(b) Ranging performance at different distance

FIGURE VI. RANGING PERFORMANCE COMPARISON AT ONE DIMENSION.

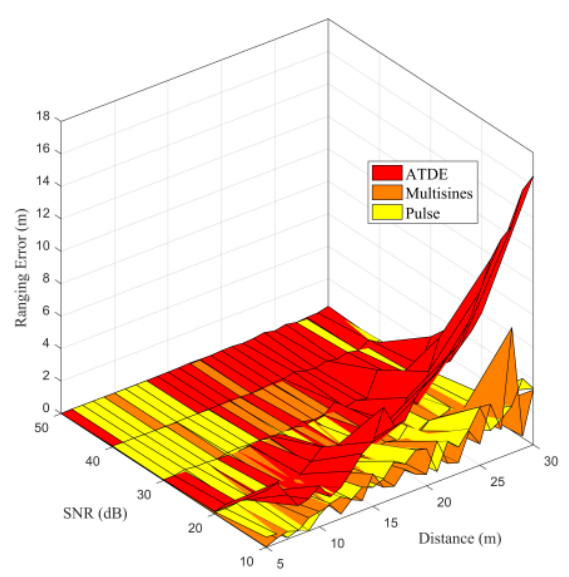

Figure VII. Ranging performance comparison at two dimension.

\section{CONCLUSION}

In this paper, we proposed a wideband signal based NFER system to improve the ranging performance. We used the frequency domain phase measurement to get the phase difference between electric field signal and magnetic field signal. Exploiting the phase differences at multiple frequencies, we use least squares method to estimate the distance. The simulation results show that the proposed wideband NFER system can reduce the ranging error at low SNR environment and within a wide range of distances. Therefore, the proposed wideband NFER system can improve the ranging performance.

\section{ACKNOWLEDGMENT}

This work is supported by National Key R\&D Program of China (2016YFB0700503) and National Natural Science Foundation of China (NSFC) project No. 6167105.

\section{REFERENCES}

[1] D. Dardari, P. Closas, and P. M. Djuric, "Indoor tracking: Theory, meth-ods, and technologies," IEEE Transactions on Vehicular Technology, vol. 64, no. 4, pp. 1263-1278, 2015.

[2] X. Tian, R. Shen, D. Liu, Y. Wen, and X. Wang, "Performance analysis of rss fingerprinting based indoor localization," IEEE Transactions on Mobile Computing, vol. 16, no. 10, pp. 2847-2861, 2017.

[3] H. G. Schantz, "A real-time location system using near-field electromagnetic ranging," in Antennas and Propagation Society International Symposium, 2007 IEEE. IEEE, 2007, pp. 3792-3795.

[4] H. G. Schantz and D. W. Langford, "Near field electromagnetic positioning system and method," Nov. 20 2007, uS Patent 7,298,314.

[5] D. Chariag, D. Guezgouz, J.-C. Le Bunetel, and Y. Raingeaud, "Modeling and simulation of temporal variation of channel and noise in indoor power-line network," IEEE Transactions on Power Delivery, vol. 27, no. 4, pp. 1800-1808, 2012.

[6] H. G. Schantz, C. Weil, and A. H. Unden, "Characterization of error in a near-field electromagnetic ranging (nfer) real-time location system (rtls)," in Radio and Wireless Symposium (RWS), 2011 IEEE. IEEE, 2011, pp. 379-382.

[7] C. Kim, F. Chin, and H. K. Garg, "Multiple frequencies for accuracy improvement in near field electromagnetic ranging (nfer)," in Personal, Indoor and Mobile Radio Communications, 2006 IEEE 17th International Symposium on. IEEE, 2006, pp. 1-5.

[8] S. Cui and F. Xiong, "Energy detection uwb system based on pulse width modulation," Journal of Engineering, 2014.

[9] C. A. Remley, N. Carvalho, D. Schreurs, and K. Gard, "Multisine signals for wireless system test and design," IEEE Microwave Magazine, vol. 9, no. 3, 2008.

[10] M. Navarro and M. Najar, "Frequency domain joint toa and doa estimation in ir-uwb," IEEE transactions on wireless communications, vol. 10, no. 10, pp. 1-11, 2011.

[11] Y. Wang, Q. Liu, and A. E. Fathy, " $\mathrm{Cw}$ and pulse-doppler radar processing based on fpga for human sensing applications," IEEE Transactions on Geoscience and Remote Sensing, vol. 51, no. 5, pp. 3097-3107, 2013.

[12] D. An, Y. Li, X. Huang, X. Li, and Z. Zhou, "Performance evaluation of frequency-domain algorithms for chirped low frequency uwb sar data processing," IEEE journal of selected topics in applied earth observations and remote sensing, vol. 7, no. 2, pp. 678-690, 2014.

[13] A. Chandra, A. Prokes, T. Mikul `a'sek, J. Blumenstein, P. Kukolev, T. Zemen, and C. F. Mecklenbrauker, "Frequency-domain in-vehicle " uwb channel modeling," IEEE Transactions on Vehicular Technology, vol. 65, no. 6, pp. 3929-3940, 2016.

[14] K. R. Rao, D. N. Kim, and J. J. Hwang, Fast Fourier TransformAlgorithms and Applications. Springer Science \& Business Media, 2011.

[15] V. Vinzi, W. W. Chin, J. Henseler, H. Wang et al., "Handbook of partial least squares," 2010.

[16] P. Wang, X. Zhang, G. Sun, L. Xu, and J. Xu, "Adaptive time delay estimation algorithm for indoor near-field electromagnetic ranging," International Journal of Communication Systems, vol. 30, no. 5, 2017 